\title{
Effects of motor activity on the elicitation and modification of the startle reflex in rats
}

\author{
JOHN R. WECKER and JAMES R. ISON \\ University of Rochester, Rochester, New York
}

\begin{abstract}
In order to examine the effects of efferent processes on the elicitation and modification of startle behavior, we administered startle-eliciting stimuli to rats while they were engaged in spontaneous motor activity. When tone bursts (Experiment 1) or electric shocks (Experiment 2) were used to elicit the reflex, its amplitude was substantially less when the rats were active than when they were quiet. Grooming, face washing, and consuming were associated with the greatest reduction. The ability of a 50-dB auditory prepulse to inhibit a subsequent auditory startle was also reduced during activity (Experiment 3 ). The amount of inhibition produced by a prepulse was decreased even when the baseline startle responses were equated in quiet and activity by varying the intensity of the eliciting stimulus, indicating that the reduction was not due to an artificial "floor effect" (Experiment 4). The study demonstrated that both sensory and motor events affect reflexive responses in the rat, as is known for the human.
\end{abstract}

Variation in the strength of an elicited reflex can be attributed in large part to the different properties of the eliciting stimulus and the conditions under which it is presented. The amplitude of the acoustic startle reflex in the rat (Fleshler, 1965) and in the human (Berg, 1974) is related to the tonal frequency of the eliciting stimulus (Foss, 1985), its intensity (Hoffman \& Searle, 1968), duration (Marsh, Hoffman, \& Stitt, 1973), rise time (Ison, 1978), and repetition rate (Davis, 1970). In addition, lawful modification of the startle reflex results from varying the intensity and lead time of sensory stimuli presented prior to or accompanying the eliciting stimulus (Hoffman \& Searle, 1968; Ison \& Hammond, 1971; Krauter, Leonard, \& Ison, 1973). Thus, elicitation and modification of the startle reflex are inherently dependent on the characteristics of external sensory inputs which temporally surround the response.

In addition to these external sensory events, variation in reflex strength appears to depend on internal efferent processes as well. In laboratory animals, stimuli paired with noxious events facilitate the startle reaction, an effect hypothesized to result from their conditioned elicitation of emotional reactions (Brown, Kalish, \& Farber, 1951; Davis \& Astrachan, 1978). In the human, Jendrassik (1883) demonstrated that when patients pulled one

This work was supported in part by a research grant from the United States Environmental Protection Agency, R-8068201, and a NSF predoctoral fellowship award to John R. Wecker. Although this research was supported by the U.S. Environmental Protection Agency, it has not been subjected to the agency's peer and policy review. Therefore, it does not reflect the view of the agency and no official endorsement should be inferred.

Portions of this paper were presented at the annual meeting of the Eastern Psychological Association, April 1981, New York. The authors wish to thank K. Sheldon and S. Rumsey for technical assistance, and A. Eastwood for editorial review. Send reprint requests to John R. Wecker, Department of Psychology, University of Rochester, Rochester, NY 14627. hand against another, concomitant knee jerks were enhanced. Other investigators, including Mitchell and Lewis (1886), Lombard (1888), and Bowditch and Warren (1890), demonstrated that a variety of self-induced muscular contractions and motor activities inhibited or facilitated the amplitude of reflexes in humans. A more recent investigation reported that systematic modification of the eyeblink reflex occurred during preparation, onset, and completion of either eyelid or forearm movements (Sanes, 1984). Thus, the expression of reflex behavior can be viewed as the result of an interaction of intrinsic reflex mechanisms with ongoing afferent and efferent processes.

Although parallel data for humans and for laboratory animals exist for stimulus modification of reflex behavior (see Hoffman \& Ison, 1980, for review), there are no reports of the general effects of concurrent motor output on reflexes in the rat. As in the human, the strength of a reflex in the rat may be dependent on concurrent efferent, as well as afferent, activity. The present investigation was a beginning approach to this question. Rats engaged in the normal range of spontaneous behaviors allowed by the test apparatus received stimuli which either elicited or modified the startle reflex. The purpose of these experiments was to record variations in the rat's startle reflex which accompany changes in the animal's ongoing activity, and to determine how afferent and efferent events interact to produce the final reflex outcome.

\section{EXPERIMENT 1}

This first experiment examined the relationship between spontaneous behaviors and the magnitude of the startle reflex elicited during these behaviors. An intense auditory stimulus was used to elicit the startle response. The amplitude of the response was recorded and the behavior of the rat at the time of stimulus presentation was classified as quiet or active, and then, if active, as one of a 
small set of behaviors. It was found that the amplitude of the startle reflex was reduced by coincident activity, and that the magnitude of this effect varied according to the type of activity.

\section{Method}

Subjects. The subjects were 11 Long-Evans hooded rats, 5 female and 6 male, born in our vivarium from Charles River stock. Their weights ranged from 300 to $350 \mathrm{~g}$ and their mean age was approximately 120 days. They were individually housed and allowed access to food and water ad lib.

Apparatus. The test cage $(8.5 \times 9.0 \times 19.5 \mathrm{~cm})$ was constructed of Plexiglas with side paneis of brass rods. During the test session the cage was placed upon a Plexiglas platform suspended across two aluminum blocks. An accelerometer (Statham Laboratories Model SA-2-300) attached to the platform directly beneath the test cage detected the force of the startle reaction. The output of the accelerometer was amplified, integrated over the $100-\mathrm{msec}$ period following the startle stimulus, and displayed as a voltage on a Fluke digital voltmeter.

The acoustic startle stimulus was a $120-\mathrm{dB}$ (re $20 \mu \mathrm{N} / \mathrm{M}^{2}$; General Radio 1561 sound level meter), $10-\mathrm{kHz}$ tone burst with a $20-\mathrm{msec}$ duration and 5-msec rise and decay times. The stimulus was produced by a Hewlett-Packard oscillator (Model 200-AB), gated through an electronic switch to a Knight stereo amplifier (Model 935) then to a General Railway Signal Company highfrequency driver (Model DWG-01095-2) located $25 \mathrm{~cm}$ from one side of the test cage. A millisecond timer controlled the duration and spacing of the stimuli. A red light bulb illuminated the test chamber, allowing visual surveillance of the subject on closed-circuit television. The experimental setup was housed inside an Industrial Acoustic Company sound-attenuating chamber lined with styrofoam panels, which was located within an Industrial Acoustic Company audiometric testing room. All control equipment was in an adjoining room.

Procedure. A test session began with placement of the rat in the test cage and the cage on the platform. Dry lab chow was placed on the floor of the cage, and the spout of a water bottle extended through the bars of the cage. Following a 5-min adaptation period, four warm-up trials were delivered in order to overcome the initial short-term habituation and achieve a stable level of responding; these trials were not included in the data analysis. Sixty presentations of the startle stimulus were then delivered at a mean intertrial interval of $30 \mathrm{sec}$. The experimenter, who was in the room containing the control and testing equipment, was alerted $3 \mathrm{sec}$ before the impending trial by a warning tone (heard only in the room containing the control equipment).

The presence and type of activity were judged at the time of stimulus presentation. It was first determined whether the subject was quiet or active. The rat was considered quiet if all four paws were on the floor of the cage, the body was oriented with the direction of the cage, and no observable movement could be detected. If the rat was active, a second evaluation classified the type of activity: consuming (eating food or drinking water); grooming (licking or scratching of the limbs or body or running the paws over the head and face); sniffing (stationary posture with observable movement of nose or vibrissae); turning (movement of the head or torso at the moment of stimulus delivery); and miscellaneous (biting the bars of the cage, teeth chattering, digging, etc.). A final category, twisting, included postural changes not acompanied by active movement. The difference between twisting and turning was that the former referred to a stationary posture other than that described for quiet, whereas the latter included movement at the time of stimulus delivery. The categories were regarded as mutually exclusive, and on each trial it was the experimenter's task to judge the predominan behavior (e.g., the animal might turn and sniff at the same time, and the experimenter judged which category best described the activity of the subject)

For the analysis of the data the responses for all 60 trials were summed and the score on each trial was expressed as a proportion $(\times 1,000)$ of that total. This normalizing procedure removed individual differences in overall reactivity so that each animal's performance was weighted equally in the analysis. The behavior of the subjects was not controlled, and, therefore, not every category of behavior was observed in all animals and scores for each type of activity were not available for every rat.

\section{Results and Discussion}

Figure I presents the mean normalized startle response for the quiet (Q), active (A), and each of the individual categories. Within each bar is the number of subjects that contributed responses to that category. The left portion of Figure 1 illustrates that the amplitude of the startle response was substantially reduced by coincident activity [Q vs. $A, t(10)=3.05, p<.05]$. The right side of Figure 1 presents the pattern of responses associated with the behavioral categories. Compared with quiet, both grooming $[t(9)=4.61, p<.01]$ and consuming $[t(3)=$ $3.76, p<.01]$ severely depressed the response. Active sniffing only slightly reduced the response compared with quiet [Q vs. SNF $t(10)=2.10, p<.10]$. Gross postural changes associated with turning or twisting did not reliably depress startle amplitude, nor did behaviors categorized as miscellaneous. However, it was noted that occasional instances of teeth chattering often resulted in an almost total depression of response amplitude.

An examination of the temporal distribution of activity revealed no differences in the occurrence of quiet, consuming, or grooming over six 10-trial blocks. In addition, no sex differences were found either in the occurrence of particular behaviors or in the distribution of activity within a session.

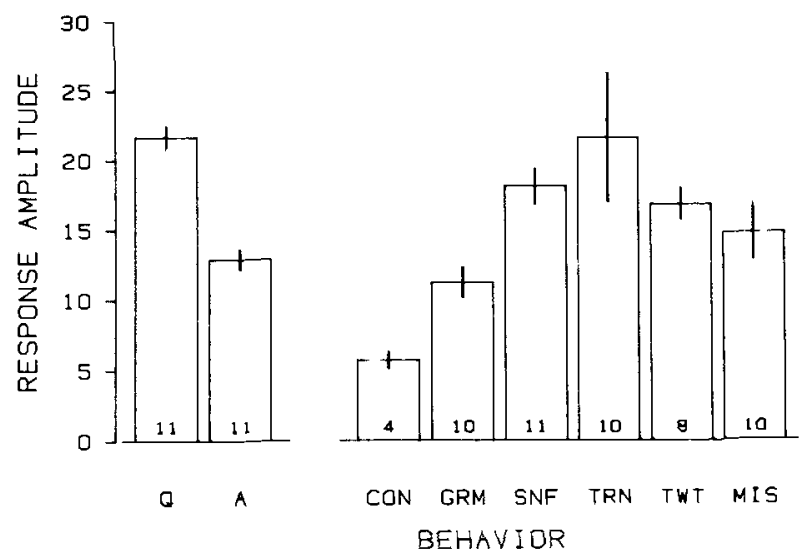

Figure 1. Mean normalized response amplitude for each behavioral category when an acoustic stimulus elicited the response. The left portion presents the average of all the responses given while the subjects were quiet $(\mathrm{Q})$ or active (A), where the active category is the mean of the categories on the right. Activity was coded as consuming (CON), grooming (GRM), sniffing (SNF), turning (TRN), twisting (TWT), or miscellaneous (MIS). The number within each bar is the number of subjects that contributed scores to that category. Error bar is \pm 1 standard error. 
The results of this experiment reveal that active rats do not respond as vigorously to a reflex-eliciting tone as do the same rats when quiet, and that the amount of this depression is dependent on the particular type of activity. Similar results have been found for young chicks (Culshaw \& Broom, 1980; Forrester \& Broom, 1980), in which probability of the occurrence of an orienting reflex was measured during ongoing activity. These authors found that the lowest probability occurred when the chicks were engaged in preening, and further, that presentation of a stimulus during the end of a bout of preening or feeding delayed the occurrence of another behavior longer than did presentation of a test stimulus early in the bout. These previous findings and the results of the present experiment indicate that the expression of reflex behavior is dependent on the ongoing behavioral activity.

\section{EXPERIMENT 2}

Since the strength of the startle response is functionally related to the intensity of the eliciting stimulus (e.g., Hoffman \& Wible, 1970), one plausible explanation for the results of Experiment 1 is that ongoing activity resulted in mechanical masking of the stimulus, effectively reducing the intensity of the acoustic stimulus. For example, an animal engaged in grooming may have blocked its pinna with its paws or body; in eating, masked the stimulus by grinding food; or in shifting the body, changed the angle of incidence of the acoustic input. One way to evaluate this explanation is to compare the results obtained in Experiment 1, in which an acoustic eliciting stimulus was used, with results obtained using a cutaneous stimulus. This procedure would eliminate the hypothesized effect of peripheral interference with the acoustic stimulus.

Cutaneous stimuli do elicit a startle response in the rat, and both cutaneous and acoustic startle are subject to the same conditions of modification by preliminary stimuli (Ash, Parisi, \& Ison, 1978). In Experiment 2, the procedure used in Experiment 1 was repeated, except that a brief electrical pulse applied to the tail was used to elicit the startle response.

\section{Method}

Twelve rats ( 8 female, 4 male) were tested. The testing and recording equipment were similar to those used in Experiment 1, except that instead of the tone a 1-mA shock was used to elicit the startle. The cutaneous stimulus was a 10-msec electrical current (dc, squarewave) generated by a constant-current shock source and delivered to the tail of the rat by perforated Grass cup electrodes (Type E56G). In this experiment, the behavioral categories of grooming and face washing were recorded separately. In addition, the category of rearing was included to compare the effects of an activity, other than face washing and grooming, that resulted in the removal of the front paws from the cage floor. All other conditions were the same as in Experiment 1.

\section{Results and Discussion}

Figure 2 presents a pattern of results very similar to that found in Experiment 1 for the auditory startle response. Responses elicited when an animal was quiet

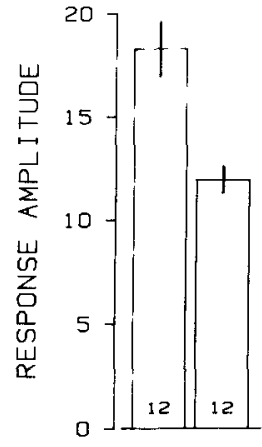

O A

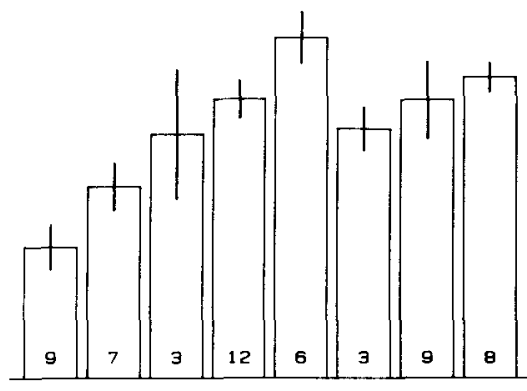

FCW GRM CON SNF REA TRN TWT MIS BEHAVIOR
Figure 2. Mean normalized response amplitude for each behavioral category when an electrical stimulus applied to the tail elicited the response. Categories are identical to those in Figure 1, except for the addition of face washing (FCW) and rearing (REA). The number within each bar is the number of subjects that contributed scores to that category. Error bar is \pm 1 standard error.

were significantly greater than responses elicited when it was active $[t(11)=4.13, p<.01]$. For the individual categories, face washing and grooming produced the greatest depression in response amplitude as compared with quiet $[t(8)=9.64, p<.01$, and $t(6)=5.88$, $p<.01$, respectively]. In addition, differences were found between quiet and sniffing $[t(11)=2.60, p<.05]$, consuming $[t(2)=9.42, p<.05]$, twisting $[t(8)=2.88$, $p<.05]$, and miscellaneous $[t(7)=2.40, p<.05]$.

These data show that the strength of the startle response elicited by electrical stimulation of the tail was also reduced during activity, and that the amount of depression was dependent on the type of coincident behavior. The similarity of these results to those seen for acoustically elicited startle reveals that response depression associated with activity operates across two sensory modalities. Thus, a simple peripheral argument based upon a mechanical blocking of the acoustic input is not sufficient to explain the results of Experiment 1.

\section{EXPERIMENT 3}

That depression of reflex activity by coincident spontaneous behavior is apparently not the result of peripheral masking of the incoming stimuli suggests that a central interaction may be occurring between the processes responsible for reflex elicitation and those responsible for motor activity. In order to examine this possibility, we introduced another event which also reduces the size of the startle response, namely, an acoustic prestimulus. The properties characterizing reflex inhibition by a prepulse have been extensively described (Hoffman \& Ison, 1980). Reflex elicitation and inhibition are generally regarded as independent events that are processed along parallel but separate channels (Ison, 1978; Ison \& Krauter, 1974; Stitt, Hoffman, \& Marsh, 1976). Combinations of separate, simultaneous stimuli across sensory modalities 
are able to produce lawful summation of inhibition (Ison, Zuckerman, \& Russo, 1975; Wecker \& Ison, in press). Therefore, the attempt to combine inhibition produced by two events, namely, activity and sensory prestimulation, may help to determine the level at which activity interferes with the response. If the two sources of inhibition summate, it is suggested that the two simultaneous events are operating independently in a manner similar to the presentation of two sensory events (Ison et al., 1975; Wecker \& Ison, in press). However, if the effect of prepulse inhibition is less evident when the animal is active, a general effect of activity on stimulus processing may be more reasonable. To test these outcomes, a prepulse of moderate intensity $(50 \mathrm{~dB})$ was presented $80 \mathrm{msec}$ prior to an acoustic eliciting stimulus.

\section{Method}

Subjects $(n=11)$ used in the previous experiment were tested approximately one month following the end of Experiment 2 . The same stimulus and response recording procedures were used except that a $20-\mathrm{msec}, 50-\mathrm{dB}, 16-\mathrm{kHz}$ tone (S1) was presented $80 \mathrm{msec}$ prior to the presentation of the $120-\mathrm{dB}, 10-\mathrm{kHz}$ startle-eliciting stimulus (S2). The prestimulus was generated by a second HewlettPackard oscillator (Model 200-AB) and was gated through an electronic switch to a McIntosh audio amplifier (Type 043-606/607). The signal was then attenuated, and delivered by another General Railway Signal Company high-frequency driver (Model DWG$01095-2$ ) located $25 \mathrm{~cm}$ from the test cage on the side opposite the speaker delivering the startle stimulus. Thirty presentations of only the acoustic startle stimulus (control trials) and 30 presentations of the prepulse followed by the startle stimulus (prepulse trials) were randomly delivered by the experimenter in a test session. Behavioral categorization was performed according to the criteria used in Experiment 1.

\section{Results and Discussion}

Figure 3 shows the mean startle response obtained following the presentation of a startle stimulus alone (control) or preceded by a weak tone pulse (prepulse) while

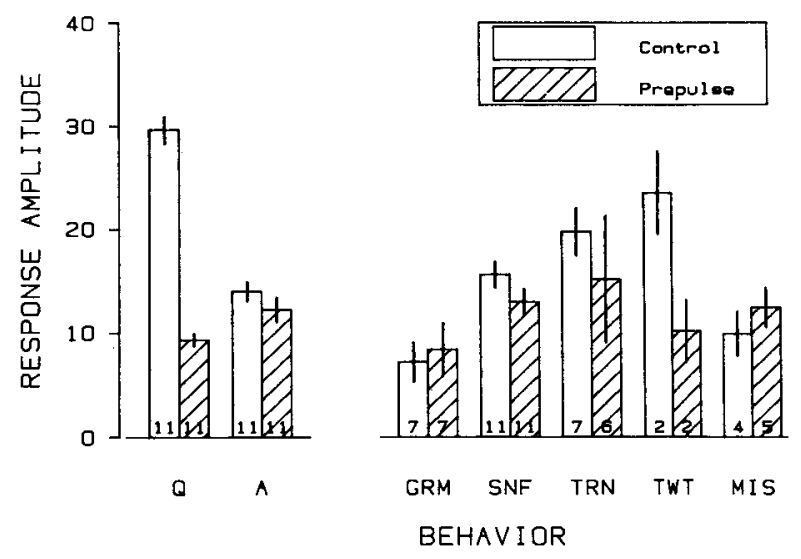

Figure 3. Mean normalized response amplitude for each behavioral category when an acoustic eliciting stimulus was presented alone (control) or when it was preceded by a 50-dB acoustic stimulus (prepulse). The number within each bar is the number of subjects that contributed scores to that category. Error bar is \pm 1 standard error. See Figure 1 for a description of the categories. the subject was engaged in spontaneous activity. Examining only the results under the control condition, it can be seen that activity reduced the size of the response by about $50 \%[t(10)=5.09, p<.01]$. For individual behaviors, the largest depression was seen for grooming $[t(6)=4.48$, $p<.01]$; responses during sniffing $[t(10)=3.58$, $p<.01]$ and miscellaneous activities $[t(3)=6.05$, $p<.01]$ also produced a depression in responding. These findings demonstrate again that activity results in a reduction in the size of the startle response, and that the strength of that depression is dependent on the particular activity of the rat at the time of stimulus presentation.

As is consistent with many previous reports, the presentation of a prepulse when the animal was quiet strongly inhibited the startle response, in this case by about $70 \%$ [control vs. prepulse while quiet, $t(10)=6.20, p<.01$ ]. This typical result is in contrast to that observed during activity, for which, although significant $[t(10)=2.26$, $p<.05$ ], the effect of the prepulse was greatly diminished (23\%). Analysis of variance revealed a significant interaction between trial type (control vs. prepulse) and behavior (quiet vs. active) $[F(3,40)=$ $32.73, p<.01$ ], which indicated that the prepulse did not inhibit the response during activity as much as during quiet. In fact, in several of the behavioral conditions, the prepulse failed to inhibit the reflex at all. These data indicate that motor activity interferes with the effect of the prepulse as well as with the eliciting stimulus.

One explanation for the finding that the inhibitory effect of a prepulse was reduced during activity is that the results were confounded by the presence of a "floor effect": activity reduced the size of the response to a point at which no further inhibition could be detected. This floor effect might mask the further reduction of the response by the combination of prepulse and activity, even if each were acting independently. Experiment 4 was designed to evaluate the possibility that a response floor may have caused the apparent decrease in inhibition during activity.

\section{EXPERIMENT 4}

In this experiment we examined the possible contribution of a "response floor" to the decreased inhibition during activity observed in the previous experiment. The problem of a floor is produced by differences in the baseline startle response: as the baseline of the control is reduced, so may potential inhibition be reduced. One approach to this problem is to compare the inhibition obtained by a prepulse when the control responses for both the quiet and active conditions are equivalent. Since the amplitude of the startle response is related to the intensity of the eliciting stimulus (Hoffman \& Searle, 1968), we attempted to match the response level for the control condition obtained during quiet to the response level for the control condition during activity by varying the intensity of the eliciting stimulus. We were then able to examine the effects of the prepulse, the activity, and the combination of the two from an equivalent baseline. 
Table 1

Mean Normalized Amplitudes in Each Behavioral Category for the Stimulus Conditions

\begin{tabular}{|c|c|c|c|c|}
\hline & \multicolumn{2}{|c|}{ Quiet } & \multicolumn{2}{|c|}{ Active } \\
\hline & $120 \mathrm{~dB}$ & $100 \mathrm{~dB}$ & $120 \mathrm{~dB}$ & $100 \mathrm{~dB}$ \\
\hline Control & 7.41 & 5.68 & 5.37 & 4.41 \\
\hline Prepulse & 3.48 & 3.00 & 4.10 & 2.99 \\
\hline
\end{tabular}

\section{Method}

In this test, 100 trials in each of four conditions were given to 12 rats ( 8 male, 4 female) over the course of two test sessions. In two of the conditions, a startle stimulus (either $120-\mathrm{dB}$ or $100-\mathrm{dB}$ ) was presented alone. In the other two conditions, the presentation of the eliciting stimulus $(120-\mathrm{dB}$ or $100-\mathrm{dB})$ was preceded by a $50-$ $\mathrm{dB}$ prepulse at an interstimulus interval of $80 \mathrm{msec}$. The apparatus, response recording, and behavioral scoring were the same as those used in the previous experiment.

\section{Results and Discussion}

In this experiment, only the categories of quiet, grooming, and sniffing were examined, because the small number of responses in the other categories spread over four conditions was not considered sufficient to provide reliable data. Sniffing failed to significantly reduce the amplitude of the response for both the 120 - and $100-\mathrm{dB}$ stimuli; therefore, all comparisons were made between quiet and grooming.

Presentation of the prepulse during quiet produced 54\% inhibition of the response to the $120-\mathrm{dB}$ stimulus and $48 \%$ inhibition of the response to the $100-\mathrm{dB}$ stimulus. In contrast, presentation of the prepulse during activity produced $16 \%[t(10)=2.67, p<.05]$ and $19 \%[t(10)=2.38$, $p<.05]$ inhibition of the responses to the 120 - and $100-$ dB stimuli, respectively. Analysis of variance revealed a significant interaction between the type of behavior and inhibition $[F(1,9)=9.41, p<.01]$, indicating that the effect of the prepulse was reduced during activity. Furthermore, the reduction in inhibition was not due to a floor effect, because although the baseline for the $100-\mathrm{dB}$ stimulus during quiet was equal to the response to the $120-\mathrm{dB}$ stimulus during activity $[t(10)=0.10$, n.s. $]$, inhibition was greatly diminished $[48 \%$ vs. $16 \% ; t(10)=2.66$, $p<.05]$. These results can be seen in the mean normalized amplitudes presented in Table 1 . Even when the mean response for the $100-\mathrm{dB}$ condition during quiet was equivalent to the response for the $120-\mathrm{dB}$ condition during activity, more inhibition was produced by the prepulse during quiet than during activity. It should be noted that percent inhibition was calculated for each subject and not from the average responses. The results of this experiment support the conclusion of the previous experiment that activity reduces the amount of inhibition produced by a sensory prestimulus, and suggest that the reduction is not due to the effect of a response floor.

\section{GENERAL DISCUSSION}

The empirical results from these experiments show unequivocally that the amplitude of a startle reflex in the rat is dependent on the activity of the animal at the time of stimulus presentation. Furthermore, face washing, grooming, and consuming were associated with the largest depression of the reflex, whereas the more labile behaviors of sniffing and turning were less effective. These two sorts of behaviors differ in a variety of dimensions, such as the vigor of motor output and, perhaps, the rhythmicity and central control with which the former are expressed (Fentress, 1976). It was also observed that postural aberrations without ongoing movement (twisting) were not consistently associated with reliable depression of the response, suggesting that active movement is necessary for amplitude reduction. Finally, response depression was seen whether an acoustic or an electrotactile stimulus was used to elicit the reflex.

One explanation for the reduction in response amplitude is that animals engaged in motor activity cannot simultaneously produce a startle response, that is, that there is competition at the final common motor pathway. However, this simple explanation is weakened by the demonstration that reflex inhibition by sensory prestimulation is also decreased during activity, even when control responding during quiet and activity are the same. Had activity simply reduced motor output associated with the startle response, then the amount of inhibition produced by the prestimulus should have been equivalent during quiet and activity (Ison \& Reiter, 1980; Stitt et al., 1976). That inhibition was also reduced during activity suggests that motor competition does not account for the reduction in response amplitude during activity.

The reduction in prepulse inhibition while the subject was active suggests a more complicated hypothesis, which is that the processing of sensory stimuli is affected during ongoing behaviors. An attenuated startle stimulus would be less effective in eliciting the reflex, and an attenuated prepulse less effective in inhibiting the response. In effect, the functional intensity of coincident sensory input may be attenuated while the organism is active (Delius, 1970). The results of some electrophysiological experiments favor the sensory attenuation hypothesis. Coulter (1974) and Coquery (1978), by examining evoked potentials in cats, showed that the emission of voluntary efferent programs produces pronounced reduction in the sensory transmission of cutaneous information along lemniscal pathways. Coquery (1978) suggested that the attenuation of incoming stimuli during ongoing activity may protect the synchrony of the motor outcome from interference by other potential demands on the system. Thus, an overall attenuation of sensory input during activity may be responsible for both the reduction in reflex amplitude and the diminution of reflex inhibition by sensory prestimulation.

\section{REFERENCES}

Ash, B. L., PARISI, T., \& Ison, J. R. (1978). Modification of acoustic and nociceptive reflexes in the rat by visual stimulation. Animal Learning \& Behavior, 6, 111-114.

BERG, K. M. (1974). Elicitation of acoustic startle in the human. (Doctoral dissertation, University of Wisconsin, 1973). Dissertation Abstracts International, 34, 5217B.

Bowditch, H. P., WARREN, J. W. (1890). The knee-jerk and its physiological modifications. Journal of Physiology, 11, 25-64. 
Brown, J. S., Kalish, H. I. , \& Farber, I. E. (1951). Conditioned fear as revealed by magnitude of startle response to an auditory stimulus. Journal of Experimental Psychology, 41, 317-327.

Coquery, J. M. (1978). Selective attention as a motor program. In J. Requin (Ed.), International symposium on attention and performance (Vol. 7, pp. 505-515). Hillsdale, NJ: Erlbaum.

Coulter, J. P. (1974). Sensory transmission through lemniscal pathways during voluntary movement in the cat. Journal of Neurophysiol ogy, 37, 831-845.

Culshaw, A. D., \& Broom, D. M. (1980). The imminence of behavioural change and startle responses of chicks. Behaviour, 73, 64-76.

DAvIs, M. (1970). Effects of interstimulus interval length and variability on startle response habituation in the iat. Joumal of Comparative \& Physiological Psychology, 72, 177-192.

Davis, M., \& Astrachan, D. I. (1978). Conditioned fear and startle magnitude: Effects of different footshock or backshock intensities used in training. Journal of Experimental Psychology: Animal Behavior Processes, 4, 791-805.

Delius, J. D. (1970). Irrelevant behavior, information processing, and arousal homeostasis. Psychologische Forschung, 33, 165-188.

FENTRESS, J. C. (1976). Dynamic boundaries of patterned behavior: Interaction and self-organization. In P. P. G. Bateson \& R. A. Hinde (Eds.), Growing points in ethology. Cambridge, England: Cambridge University Press.

FLESHLER, M. (1965). Adequate acoustic stimulus for startle reaction in the rat. Journal of Comparative \& Physiological Psychology, 60, 200-207.

Forrester, R. C., \& Broom, D. M. (1980). Ongoing behaviour and startle responses of chicks. Behaviour, 73, 51-63.

Foss, J. A. (1985). The expression of a sound's intensity through the elicitation and modification of the startle reflex in young and old rats. (Doctoral dissertation, University of Rochester, 1984). Dissertation Abstracts International, 45, 3106B.

Hoffman, H. S., \& Ison, J. R. (1980). Reflex modification in the domain of startle: I. Some empirical findings and their implications for how the nervous system processes sensory input. Psychological Review, 87, 175-189.

Hoffman, H. S., \& SeARLE, J. L. (1968). Acoustic and temporal factors in the evocation of startle. Journal of the Acoustical Society of America, 43, 269-282.

HofFMAN, H. S., \& WiBLE, B. L. (1970). Role of weak signals in acoustic startle. Joumal of the Acoustical Society of America, 47, 489-497.
Ison, J. R. (1978). Reflex inhibition and reflex elicitation by acoustic stimuli differing in abruptness of onset and peak intensity. Animal Learning \& Behavior, 6, 106-110.

Ison, J. R., \& HAMMOND, G. R. (1971). Modification of the startle reflex in the rat by changes in the acoustic and visual environments. Journal of Comparative \& Physiological Psychology, 75, 435-452.

Ison, J. R., \& KraUter, E. E. (1974). Reflex eliciting stimuli and the refractory period of the acoustic startle reflex in the rat. Journal of Comparative \& Physiological Psychology, 86, 420-425.

Ison, J. R., \& ReITER, L. A. (1980). Reflex inhibition and reflex strength. Physiological Psychology, 8, 345-350.

Ison, J. R., Zuckerman, M., \& Russo, J. M. (1975). Combination rules for inhibitory stimuli. Journal of Experimental Psychology: Animal Behavior Processes, 1, 318-325.

JENDRASSIK, E. (1883). Beitrage zur Lehre von den Sehnenreflexen. Deutsches Archiv für Klinische Medizin, 33, 177-199.

Krauter, E. E., Leonard, D. W., \& Ison, J. R. (1973). Inhibition of the human eyeblink by a brief acoustic stimulus. Journal of Comparative \& Physiological Psychology, 84, 246-251.

LOMBARD, W. (1888). The variations of the normal knee-jerk and their relation to the activity of the central nervous system. American Journal of Psychology, 1, 2-71.

Marsh, R. R., Hoffman, H. S., \& STITT, C. L. (1973). Temporal integration in the acoustic startle reflex of the rat. Journal of Comparative \& Physiological Psychology, 82, 507-511.

Mitchell, S. W., \& LEWIS, M. J. (1886). Physiological studies of the knee-jerk, and of the reactions of muscles under mechanical and other excitants. The Medical News (Philadelphia), 48, 169-173.

SANES, J. N. (1984). Voluntary movement and excitability of cutaneous eyeblink reflexes. Psychophysiology, 21, 653-664.

Stitt, C. L., Hoffman, H. S., \& Marsh, R. R. (1976). Interaction vs. independence of startle modification processes in the rat. Journal of Experimental Psychology: Animal Behavior Processes, 2, 260-265.

WECKER, J. R., \& IsON, J. R. (in press). Visual function measured by reflex modification in rats with inherited retinal dystrophy. Behavioral Neuroscience.

(Manuscript received January 6, 1986; revision accepted for publication May $1,1986$. ) 